\title{
Development of an Android Based SCADA System for Induction Motor Application
}

\author{
Patrick O. Oseghale and Nkwachukwu Chukwuchekwa
}

\begin{abstract}
Majority of SCADA (Supervisory Control and Data Acquisition) systems that is being deployed and implemented all over the world utilizes a much larger network that is complex and expensive. This paper provides and demonstrates a cheaper alternative. The developed system which may be used for domestic applications has the ability to monitor the supply voltage, speed, casing and stator temperature as well as to control the speed of the motor. It can transmit the data of these monitored parameters, wirelessly, between the base station (site) and mobile station (android phone) through Bluetooth. The mobile station serves as the display medium to enable the user to view these parameters and also have input keypads that the user can use to alter the speed. To achieve this, an embedded system was used which involves the programming of PIC microcontrollers using assembly language. Other discrete components such as resistor, capacitor, transistors etc. were also utilized. A variable frequency driver was designed to drive the induction motor and interface with the microcontroller. After the design, construction and testing, the results were satisfactory as the SCADA system could alter induction motor speeds, collate motor casing and stator temperature, supply voltage and speed in revolutions per minute (RPM) and transmit them to the android phone. Temperature range of $24^{\circ} \mathrm{C}$ to $51^{\circ} \mathrm{C}$, motor speed of 747 to $2234 \mathrm{RPM}$, and supply voltage of 179 to $221 \mathrm{~V}$ AC was measured.
\end{abstract}

Keywords - Android, Bluetooth, PIC, SCADA, Induction Motor.

\section{INTRODUCTION}

Production and manufacturing processes are usually complex as they involve numerous stages and processes that can be as much as 1000 processes or more. These processes are usually tagged industrial processes or activities and they cannot be handled manually due to the obvious disadvantages of slow speed, human errors and problems like absenteeism, etc., hence the need for automated control systems to help industries meet up with the demand for modern production activities for improved productivity and profitability.

Control consists of monitoring the state of a critical parameter, detecting when it varies from the desired state, and taking action to restore it. Control can be discrete or analog, manual or automatic, and periodic or continuous [1]. It is impossible to keep control and supervision on all industrial activities manually, hence some automated tool is required which can control, supervise, collect data, analyses data and generate reports [2].

Globally, societies in their daily endeavors have become so dependent on automation that it is difficult to imagine life without automation systems. In addition to the industrial production with which it is popularly associated, it now covers a number of unexpected areas. Trade, environmental protection engineering, traffic engineering, agriculture, building engineering, and medical engineering are but some of the areas where automation is playing a prominent role. This automation control picture has changed since computers and software have made their way into every component and element of communications and automation [3].

SCADA (Supervisory Control and Data Acquisition) control systems and other control systems platforms like distribution control system (DCS) were developed to help in control and management of industrial processes. SCADA is an industrial computer-based control system employed to gather and analyze the real-time data to keep track, monitor and control industrial equipment in different types of industries. SCADA is used to manage any kind of equipment. Typically, SCADA systems are used to automate complex industrial processes where human control is impractical - systems where there are more control factors, and more fast-moving control factors, than human beings can comfortably manage [4].

SCADA systems became popular in the 1960's as the need to monitor and control remote gear grew. Early systems were built from mainframe computers and required human oversight to operate. Today with new technology, SCADA systems are much more automated. SCADA systems around the world find use in a wide variety of applications. Electric power generation, transmission and distribution are examples of such industry. Electric utilities use SCADA systems to detect current flow and line voltage, to monitor the operation of circuit breakers, and to take sections of the power grid online or offline. Another is the water and sewage industry. State and municipal water utilities use SCADA to monitor and regulate water flow, reservoir levels, pipe pressure and other factors. Buildings, facilities and environments where specific temperature and humidity considerations are important are other applications. Facility managers use SCADA to control HVAC, refrigeration units, lighting and entry systems [5]. The manufacturing industry uses SCADA systems to manage parts inventories for just-in-time manufacturing, regulate industrial automation and robots, and monitor process and quality control [6].

SCADA is not a specific technology, but a type of function. SCADA by virtue of its definition includes any device that gets data about a system in order to control that system. It is basically a system for remote monitoring and control that operates with coded signals over communication channels. The system may be combined with a data 
acquisition system by adding the coded signals over communication channels to acquire information about the status of the remote equipment for display or for recording functions. It is a type of industrial control system [7].

An industrial process involves a lot of stack protocols perfectly lined to serve a distinct function. It involves, in some cases, automation and firm control. The process to be controlled may be mechanical, electronic or chemical processes all well arranged to produce a specific desired purpose. Machines and equipment may not be the only thing that may need artificial control protocols as other forms of environmental status conditions may also need to be artificially controlled. Such as the light intensity of an environment, the atmospheric pressure, the temperature, environmental humidity etc.

Usually, these controls can be in the form of manual methodology which would involve the on-site presence of the controlling personnel to physically push the buttons to initiate the control process that will eventually control the equipment or devices that is desired to be controlled, or in some cases, these controls would be done wirelessly but they also require the physical presence of the control personnel to have eyes-on-the-job supervisory abilities to ensure that all the wireless commands that were given are actually carried out by the operating machines [7].

Meanwhile in the other forms of control where by environmental conditions are desired to be set, conditions such as the temperature, light intensity, pressure, humidity etc., environments conditional altering equipment would be employed. This is sometimes referred to as a secondary control process because it involves the use of a device or process to control another process. For instance, in the temperature regulation of an environment, the use of an air conditional unit or a simple domestic fan would be employed.

In all the above mentioned control process, the acquisition of data may become necessary. Personnel could for instance, needs to know the status of a particular machine to ensure that the total process would not be flawed. This is important because processes usually employ handshake ability in its structure. This means that the synchronous working of all the machines or equipment in the total process is extremely important for the process to be successful. These data could also be important on the environmental conditioning process as well which involves the adjustment of physical environmental conditions such as light intensity, atmospheric pressure, relative humidity etc. Data acquisition of the status of these environmental conditions may also be very important to ensure a smooth operation of a process. The accumulated data could be desired to be manipulated, analyzed, stored or just displayed on a screen. Usually, the screen is similarly placed on site and the supervising personnel have to be physically present to view the display and make further interpretation or analysis if desired [7].

However, there exist methodologies whereby control of industrial equipment, machines or processes can be done over a wireless prioritized communication channel network system. In this form of operation, machines operations can be controlled wirelessly using the communication network as well as the monitoring of the status of the machine and other parameters such as its speed, its temperature and a whole lot more. Also environmental conditions can be monitored remotely too. The acquired data from the site from all these parameters would also be transmitted wirelessly via the same communication network, to a remote site for viewing or further manipulation or analysis. This is the basis for a SCADA system.

There are no simple SCADA units in the market as SCADA systems are used in industries and as such are not cheap or cost-effective for small scale usage. Hence this work is aimed at designing a small scale SCADA control system that can control and monitor one or two inputs and outputs with keypad user input and LCD display as a cost effective design for small scale use and implementation.

The SCADA design implemented in this work is capable of controlling the speed of an induction motor and monitor casing temperature, supply voltage and speed from a remote location and view obtained data from the site, on a screen placed several meters away.

\section{CONCEPT OF THE DEVElOPED SCADA SYSTEM}

\section{A. Mobile Station (Android Phone)}

This refers to the android phone serving as the mobile station as it has its own display and keypad on it whereby all controls that were possible on any permanent base station can also be implemented with the help of the designed application. This in comparison with the supervisory computer is more flexible as the user can be anywhere within the network and still communicate with the site.

\section{B. Android}

Android is a mobile operating system developed by Google, based on a modified version of the Linux kernel and other open source software and designed primarily for touch-screen mobile devices such as smart-phones and tablets [8]. This is the Human Machine Interface (HMI) used which is the Graphic User Interface (GUI) supporting the SCADA application.

\section{Bluetooth}

Bluetooth is a standardized protocol for sending and receiving data via a $2.4 \mathrm{GHz}$ wireless link. It's a secure protocol, and it's perfect for short-range, low-power, lowcost, wireless transmissions between electronic devices.

These days it feels like everything is wireless, and Bluetooth is a big part of that wireless revolution. Bluetooth is found embedded into a great variety of consumer products, like headsets, game controllers. In the world of embedded electronics hackery, Bluetooth serves as an excellent protocol for wirelessly transmitting relatively small amounts of data over a short range $(<100 \mathrm{~m})$. It's perfectly suited as a wireless replacement for serial communication interfaces.

The Bluetooth protocol operates at $2.4 \mathrm{GHz}$. There is a standardized set of rules and specifications that differentiates it from other protocols. The characteristics of Bluetooth include a frequency band that ranges from 2.4 $\mathrm{GHz}$ to $2.483 \mathrm{GHz}(83 \mathrm{MHz}$ ). Bluetooth devices may have different RF power classification levels. The power versions of Bluetooth include; $1 \mathrm{~mW}$ - class 3, $2.5 \mathrm{~mW}$ - class 2, and $100 \mathrm{~mW}$ - class 1 . More details are given in table 1 below. 


\begin{tabular}{|l|l|c|}
\multicolumn{3}{|c}{ TABLE I: BLUETOOTH RF POWER CLASSES [9] } \\
\hline Class & $\begin{array}{l}\text { Maximum permitted power } \\
(\mathrm{mW} / \mathrm{dBm})\end{array}$ & Range(approximate) \\
\hline Class 1 & $100 \mathrm{~mW}(20 \mathrm{dBm})$ & 100 meters \\
\hline Class 2 & $2.5 \mathrm{~mW}(4 \mathrm{dBm})$ & 10 meters \\
\hline Class 3 & $1 \mathrm{~mW}(0 \mathrm{dBm})$ & 1 meter \\
\hline
\end{tabular}

The key technology used in the Bluetooth system include frequency hopping spread spectrum - FHSS. Frequency hopping spread spectrum is a radio transmission process where a message or voice communications is sent on a radio channel that regularly changes frequency (hops) according to a predetermined code. Bluetooth specification 1.2 introduced adaptive frequency hopping - AFH that can reduce the effects of interference between Bluetooth and other types of devices. AFH adapts the access channel sharing method so that the transmission does not occur on channels that have significant interference. By using interference avoidance, devices that operate within the same frequency band and within the same physical area can detect the presence of each other and adjust their communication systems to reduce the amount of overlap (interference) caused by each other. Bluetooth was used as the communication infrastructure between the user and the site; instead of wired communication, wireless was implemented.

\section{PIC Microcontroller}

Instead of PLCs, PIC (Programmable Intelligent Computer) was used in the development. PLCs are bigger than PICs. PICs are simpler to program, hence an improvement.

Peripheral Interface Controller or Programmable Interface Controller or Programmable Intelligent Computer is a small computer on a single integrated circuit. They are designed for embedded applications [10]. It consists of CPU, RAM, ROM, timers, and counters, etc. PIC microcontroller are the world's smallest microcontrollers. They can be found in phones, embedded systems, computer control systems and alarm systems [11]. For this project, the controller for the communication stage is PIC microcontroller (PIC16F876A). The microcontroller generates the programmed frequency rate, drives the VFD stage to condition the voltage level to appropriate level. PIC microchips are designed with Harvard architecture, and are offered in various device families. The baseline and mid-range families use 8 - bit wide data memory, and the high end families use 16-bit data memory. Instruction word is in the size of 14-bit for the PIC16. Almost all type of PIC microcontroller is included in a class of 8-bit microcontrollers of RISC architecture. Basically, the PIC architecture is minimized to be a simpler item but it still operates at the same function.

\section{MATERIALS AND MethodS}

The design consists of the following stages:

1) Power supply stage.

2) Sensor and transducer.

3) Communication interface stage.

4) Variable frequency drive.

5) Communication interface.

The specifications for the design are as listed below:

1) The power source is a mains supply of $220 \mathrm{~V}$.
2) Frequency range of induction motor is $30 \mathrm{~Hz}$ to $80 \mathrm{~Hz}$.

3) The communication link is Bluetooth.

4) The mobile station is an android phone.

\section{A. Power Supply Design}

The circuit required a DC power supply capable of supplying a regulated $+12 \mathrm{~V}$ and $+5 \mathrm{~V}$ for the control circuit and the dc supply for the H-bridge drive circuit of the variable frequency drive.

The step-down transformer of $220 \mathrm{~V}$ to $24 \mathrm{~V}$ AC was used. The 24V AC was used for the power supply and also used for supplying voltage sensing. The control circuit was expected to use lesser than $200 \mathrm{~mA}$ but was chosen as worst case value and as such a $500 \mathrm{~mA}$ transformer was used. This $24 \mathrm{~V}$ AC voltage was rectified to DC by a bridge rectifier. The capacitors serve as filter capacitors.

Output Secondary Voltage $=24 \mathrm{Vrms}$.

Peak Voltage $V p=\sqrt{2} \times V r m s$

$$
=24 \mathrm{x} \sqrt{2}=33.9 \mathrm{~V}
$$

Peak output voltage from bridge Rectifier

$V p . r=V p-2 V d$

$$
\begin{aligned}
& =33.9-2(0.7) \\
& =33.9-1.4 \\
& =32.5 \mathrm{~V}
\end{aligned}
$$

A medium sized diode $1 \mathrm{~N} 4004$ was used in the design to realize the bridge rectifier and has the following specifications:

1) Output Current is $1 \mathrm{~A} @ 50 \mathrm{oC}$

2) I surge $(\max )$ is $100 \mathrm{~A}$

3) Reverse voltage $\left(V_{R R M}\right)$ is $400 \mathrm{~V}$

The following expression was used to determine capacitance.

Ripple Voltage $V r=I o \div 2 F C$

Where Io $=$ Output Current, $\mathrm{F}=$ Frequency

For Io $=0.2 A, V r=1 \mathrm{~V}$

$$
\begin{aligned}
\mathrm{C} 1 & =\frac{0.2}{2 \times 50 \times 1} \\
\mathrm{C} 1 & =0.002 \mathrm{~F} \\
& =2000 \mu \mathrm{F}
\end{aligned}
$$

This is a capacitor with at least $2000 \mu \mathrm{F}$ and working voltage of $33.9 \mathrm{~V}$ is needed. The one chosen for the design is $35 \mathrm{~V} 2,200 \mu \mathrm{F}$.

$\mathrm{C} 2$ is an optional capacitor but put in place to remove any residual $\mathrm{AC}$ ripples from the supply and noise from the circuit operation and the chosen value is $25 \mathrm{~V}, 10 \mu \mathrm{F}$.

A voltage regulator of 7812 and 7805 was used in the design to obtain the regulated $+12 \mathrm{~V}$ and $+5 \mathrm{~V}$ respectively. The specifications are as shown:

7812 Voltage Regulator

Maximum Input Voltage: $35 \mathrm{~V}$

Output Voltage: $12 \mathrm{~V}$

Drop out Voltage: 2V

Operating temperature: $0^{\circ} \mathrm{C}-150^{\circ} \mathrm{C}$

7805 Voltage Regulator

Maximum input voltage: $35 \mathrm{~V}$

Output voltage: $5 \mathrm{~V}$

Drop out voltage: $2 \mathrm{~V}$

Minimum input voltage: $7 \mathrm{~V}$ 
Output current: $1 \mathrm{~A}$

The supply for H-bridge drive involves direct rectification of mains 220V AC to DC. Diodes D5 to D8 make up the bridge rectifier while capacitor $\mathrm{C} 3$ is the filter capacitor.

Mains voltage $V r m s=220 \mathrm{Vrms}$

Peak Voltage $V p=220 \times \sqrt{2}=311 \mathrm{~V}$

Peak output voltage from bridge Rectifier

$V p r=V p-2 V d$

$$
=311-2(0.7)=311-1.4=309.6 \mathrm{~V}
$$

An I.C bridge Rectifier QL62F6010W was used in the design and has the following specifications:

1) Output Current is 60A @ $50^{\circ} \mathrm{C}$

2) Current surge $(\max )$ is $200 \mathrm{~A}$

3) Reverse voltage $\left(V_{R R M}\right)$ is $1000 \mathrm{~V}$

\section{B. Design of the Sensor and Transducer Stage}

The sensors and transducers in this work serve to interface the physical quantities and parameters with the circuit in the form of voltage and current. In this work sensors used were for temperature measurement of induction motor casing and stator coil, tachometer for speed measurement and supply voltage using voltmeter.

\section{Casing and Stator Casing Temperature}

The temperature sensor chosen for the design for measuring stator and casing temperatures is the popular LM35 IC temperature sensor. LM35 is a three terminal integrated circuit temperature sensor as shown in figure 3.2 that gives a linear voltage output of $10 \mathrm{mV}$ per degree Celsius. Available in two versions, one operating from $0 \mathrm{oC}$ to $100 \mathrm{oC}$ (DZ version) the other is from $-40 \mathrm{oC}$ to $+100 \mathrm{oC}$ (CZ version). The $\mathrm{DZ}$ version was used in this work.

\section{Voltmeter}

A DC supply was used for AC voltage sensing and level measurement while a voltage divider was used to scale the value to accurate reading range. The $\mathrm{AC}$ voltmeter gets its supply from the step-down $24 \mathrm{~V}$ transformer.

Tachometer

This stage use infra-red LEDs for speed detection for rotation and running measurement. It consists of a transmitter and receiver infra-red LED unit. The system uses the beam reflection by a dark white color difference on the shaft of the motor. For transmitter/emitter there is a limiting resistance. The infra-red TIL32 LED specifications are:

1) Forward voltage drop $\mathrm{Vd}$ is $2.5 \mathrm{~V}$

2) Forward current Ic is $15 \mathrm{~mA}$

$R 1=\frac{V s-V d}{I c}$

$$
=\frac{5-2.5}{15 \times 10^{-3}}=166 \Omega
$$

\section{Communication Interface Stage}

The communication interface stage consists of a Bluetooth module and a microcontroller. The Bluetooth module was used to establish communication link between the site and the mobile station. The microcontroller functions is to communicate with the sensors to gather their outputs voltage for the quantity measured, process them and communicates it to the mobile station. It also receives control data for speed control and communicates it to the variable frequency drive stage.

\section{Variable Frequency Drive Stage}

The variable frequency drive section deals with the power drive of the induction motor. It does this using frequency drive technique to alter the speed of an induction motor. It consists of a microcontroller and an H-bridge driver stage.

\section{E. Control Program and Algorithm}

The program for the circuit was written in assembly language. The assembler used was MPLAB 7.40. The codes were compiled by this compiler and downloaded to the PIC16F876A used for the communication interface stage. Fig. 1 shows the flowchart of the code development. The second microcontroller PIC16F84 handling the pulse drive has its code development flowchart and algorithm is in Fig. 2 .

Referring to Fig. 1, when there is data received from the mobile station to the site through the Bluetooth module, the microcontroller parses the data to extract the speed data which is processed and sent to the microcontroller of the variable frequency drive stage but in the absence of that it would normally take the analog output of the temperature sensors and convert them to digital including speed pulses from the tachometer, process them and transmit the data to the mobile station.

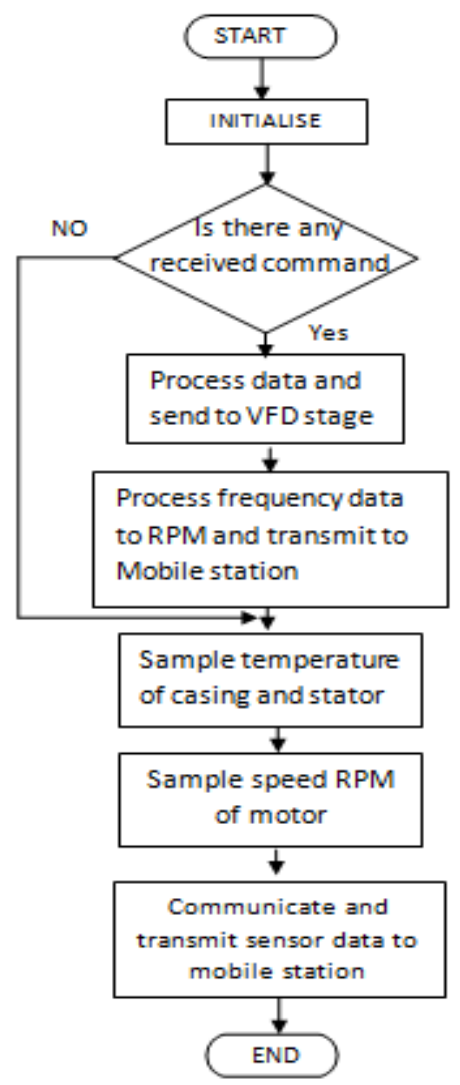

Fig. 1. Flowchart for codes development for microcontroller communication interface stage.

Referring to Fig. 2, the microcontroller first waits for data from the microcontroller of the communication interface stage. When it receives data, it will extract the frequency data, convert it to pulse width of the frequency i.e. period and use that as the pulse width of the pulses generated to 
drive the MOSFETS.

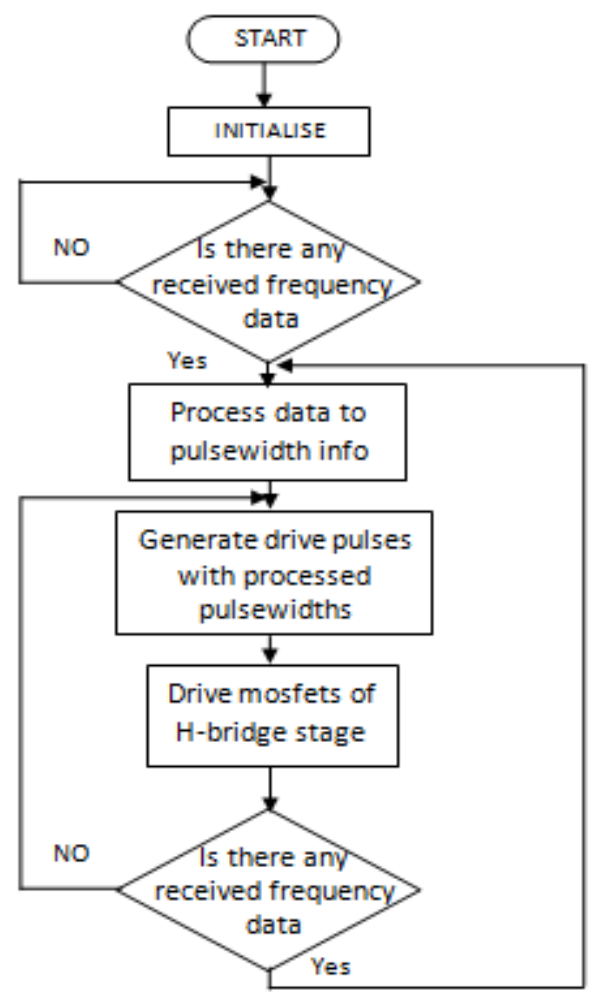

Fig. 2. Flowchart of the microcontroller of VFD stage.

\section{Mobile Station}

The mobile station here refers to the android phone with a HyperTerminal program for communicating with the site system through Bluetooth. The Bluetooth RS232 Terminal app was installed from Google play store. It can interface with any Bluetooth device and establish serial communication link based RS232 protocol which the HC-06 module uses.

When the user opens the app, it will automatically show the received information transmitted by the site station of temperature, supply voltage and speed RPM. It has the field for the user to enter the frequency for the speed required and send to the site. Fig. 3 shows the block representation of the various subsystems or stages, showing the relationship between them.

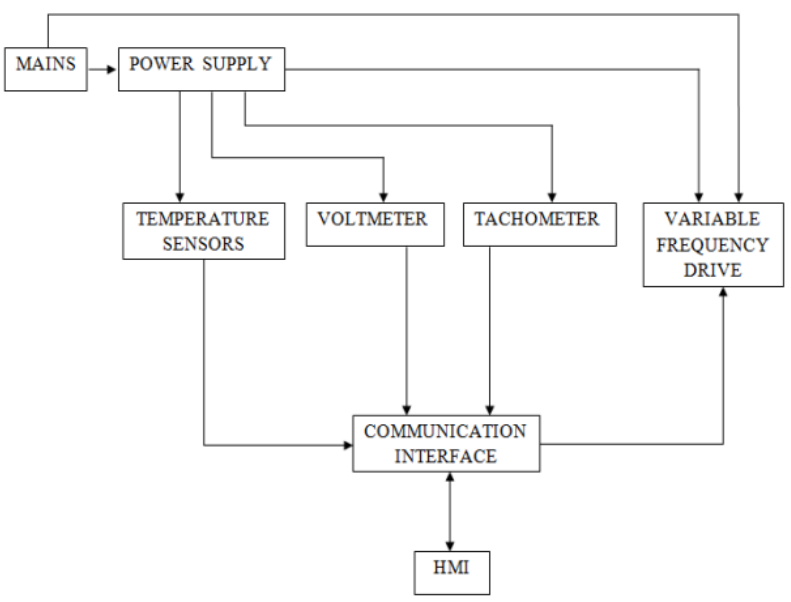

Fig. 3. Block diagram of the subsystems or stages.

\section{RESULTS AND DISCUSSION}

After the design and construction was done, there was need to carry out tests to prove the working capabilities. Some tests including temperature, speed, voltage sensing and frequency tests were carried out. Fig. 4 to Fig. 7 shows the results that were gotten from these tests.
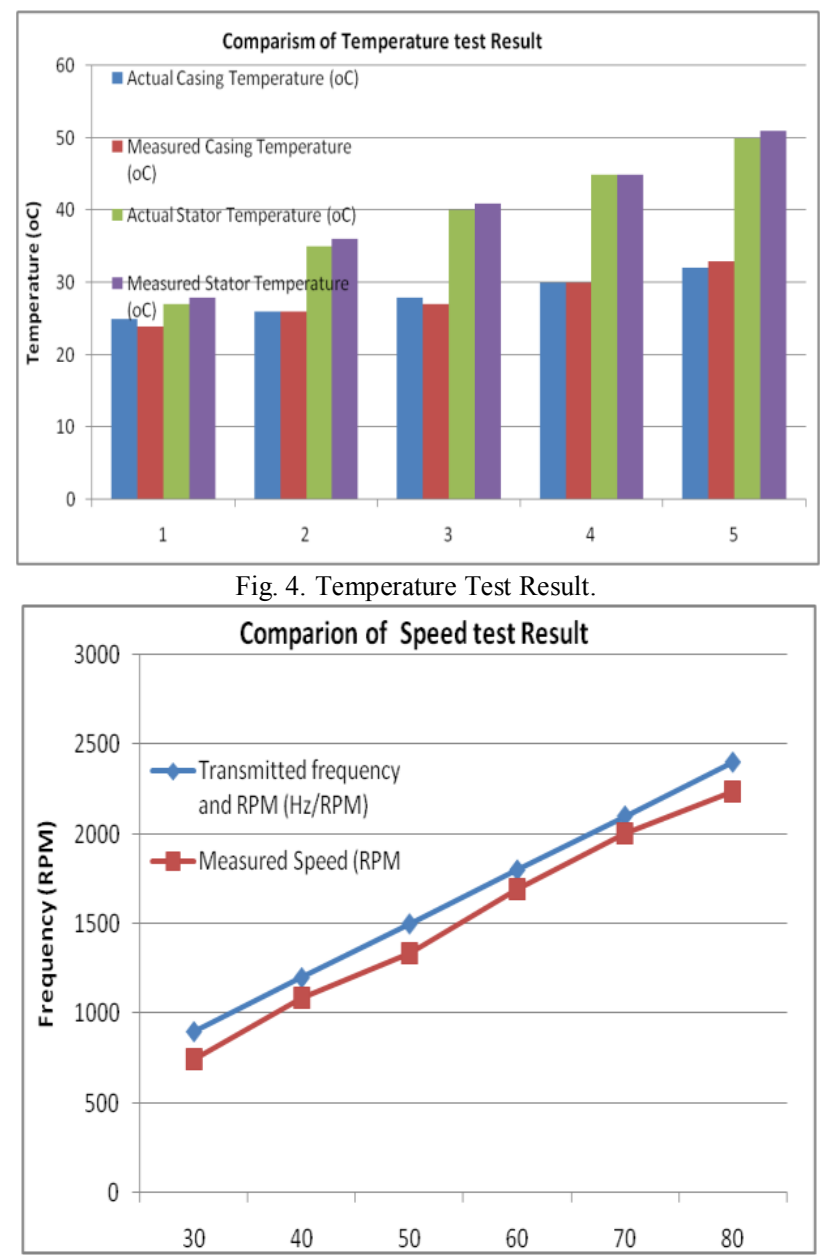

Fig. 5. Speed test result.

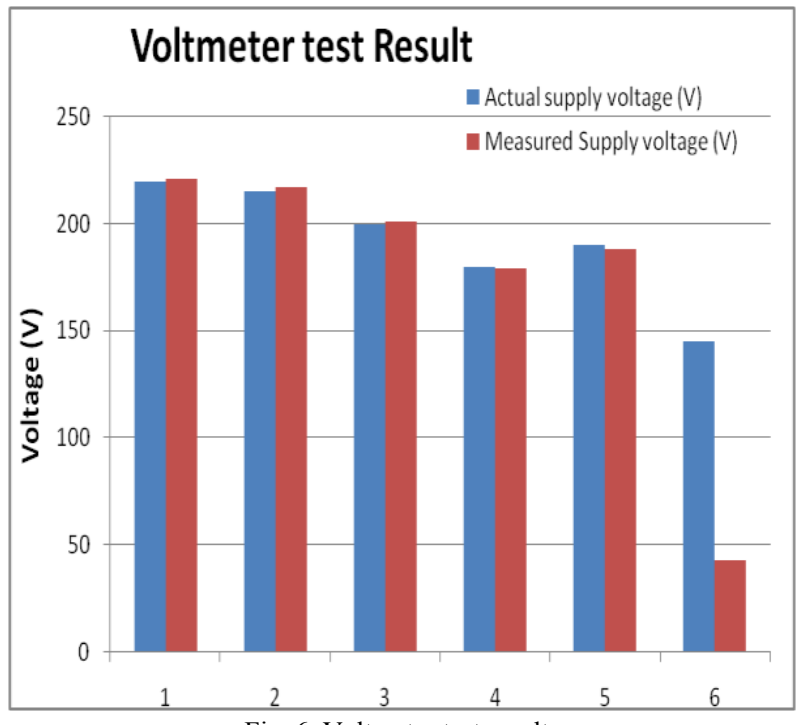

Fig. 6. Voltmeter test result. 


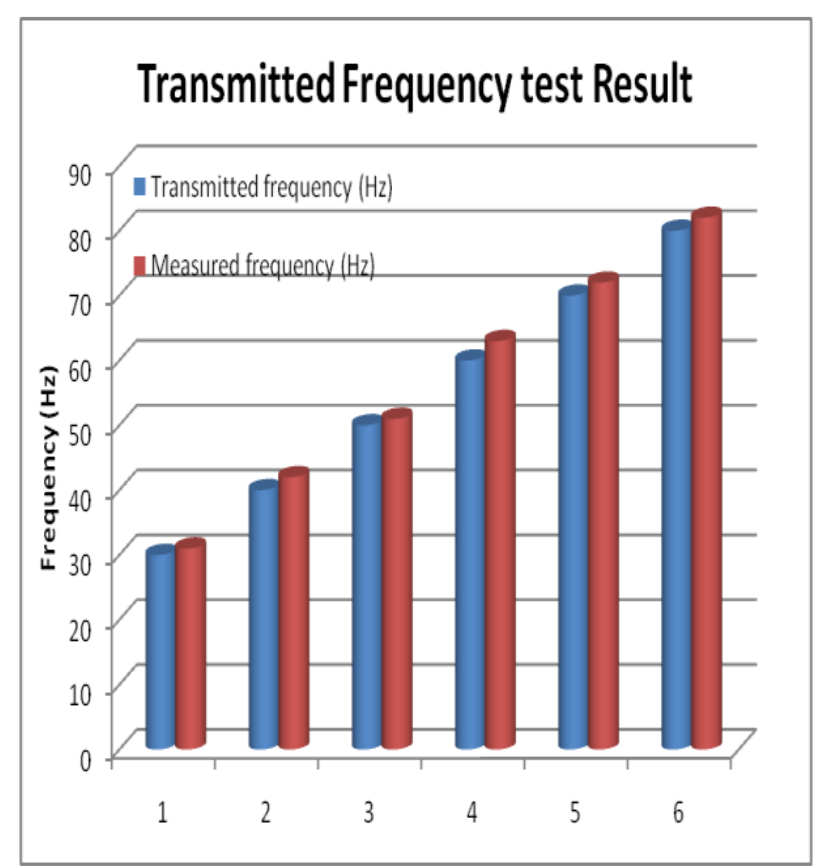

Fig. 7. Transmitted frequency test result.

The test result of Fig 4 shows that the system can measure the temperatures from the casing and stator temperature sensors. The test was done using a digital thermometer to compare and verify the values. A digital thermometer is a device used for measuring temperature that are easily portable, have permanent probes and a convenient digital display.

The casing temperature was first examined. This was done by placing both the LM35 DZ sensor and the digital thermometer probe just outside the body of the AC induction motor. After this, the motor was then started. As it was working, heat was generated which was measured by both the LM35 DZ and the digital thermometer simultaneously in real time. The values gotten were similar. It could be seen that measurement points 2 and 4 had the same readings but the rest varied with just $\pm 1^{\circ} \mathrm{C}$. The results were satisfactory.

The stator temperature was further examined with the digital thermometer and the LM35 DZ sensor simultaneously in real time. They were placed inside the casing just close to the stator and the readings were taken. From Fig. 4, the results were very close with just $\pm 1^{\circ} \mathrm{C}$ difference except measurement point 4 that has the same values. The results were also satisfactory.

Fig. 5 shows the result of the speed test. Ideally, a standard frequency of $50 \mathrm{~Hz}$ will give $1500 \mathrm{rpm}$. Practically this is not so as changes are experienced due to factors like unstable voltage and additive white Gaussian noise (AWGN) which is a sum of all the individual noise generated by electronic components. Factors like these can cause fluctuations in the values of the result and would not bring about an ideal revolution per minute. As different frequency values were transmitted from the android phone to the system, speed changes were seen and the information was passed on to the android phone with the help of the tachometer through the communication interface. The results from this information were then noted as seen in the figure. Conclusively, it shows that the system can measure the speed and from observation the supply voltage was also affecting the speed which causes deviations from the standard speeds and frequency relationship. Other notable observation is the presence of harmonics as the motor is being driven by pulse waveform.

The essence of the voltmeter is for the user to know the AC voltage value that is entering the system from the mains supply. From Fig. 6, it can be noted that the test was done by monitoring the supply fluctuation over a period from the output of different phases of the utility mains in order to have a wider range of voltage values. The actual supply voltage values were read with the help of a multimeter. The values were noted simultaneously with the displayed values on the screen of the android phone.

The PIC16F876A microcontroller of the communication interface was programmed to display "POOR SUPPLY" anytime the mains supply voltage falls below $150 \mathrm{~V}$. In order to test it, getting a voltage as low as $<150 \mathrm{~V}$ was made possible with the help of a $1 \mathrm{KVA}$ generator and getting its power reduced. This was added as a safety or warning feature to bring to the attention of the user that the supply voltage going into the system was now very low. For more alertness, it is the only display shown to the user. The results were satisfactory.

The result shown in Fig. 7 was achieved by using a frequency meter to compare and verify the frequency value that is being transmitted. The figure shows that the device is actually doing frequency variation as shown from the readings and there is communication between the mobile station and the site through the Bluetooth.

\section{CONCLUSION}

The work was undertaken to demonstrate the basic functions of a SCADA system on a small scale. The system can monitor a process, effect control and acquire data which is transmitted to the mobile station. This was applied for control and supervision of an induction motor. The system was designed to drive an induction motor of choice. Each speed sequence was monitored using its attached tachometer front end.

The mobile station which is an android phone has the human machine interface and GUI to send and monitor data and tests results shows the system worked as expected. The system design and tests carried out after construction showed that the device worked. The circuit when tested worked satisfactorily as it was able to drive the induction motor and adjust the speeds to each speed transmitted control set values.

\section{REFERENCES}

[1] DEPARTMENT OF THE ARMY Washington DC, "Department of the Army, TM 5-601, Supervisory Control and Data Acquisition (SCADA) Systems for Command, Control, Communications, Computer, Intelligence, Surveillance, and Reconnaissance (C4ISR) Facilities," 2006.

[2] B. Raman, "An Introduction To SCADA For Electrical Engineers Beginners," Electrical Engineering Portal, 2013. [Online]. Available: electrical-engineering-portal.com/an-introduction-to-scada-forelectrical-engineers-beginners. [Accessed: 08-May-2018].

[3] IDC Technoogies, Pocket Guide on Industrial Automation For Engineers and Technicians, 1st ed. 2007.

[4] EDGEFX, "Know all about SCADA Systems Architecture and Types with Applications," 2016. [Online]. Available: 
www.edgefxkits.com/blog/scada-system-architecture-types-

applications. [Accessed: 06-Jul-2018].

[5] C. Queiroz, A. Mahmood, and Z. Tari, "SCADASimA Framework for Building SCADA Simulations," IEEE Trans. Smart Grid, vol. 2, no. 4, pp. 589-597, 2011.

[6] DPS Telecom, "SCADA Introduction Fundamental Implementation," 2016. [Online]. Available: www.dpstele.com/scada/introductionfundamentals-implementation.php. [Accessed: 01-Jun-2018].

[7] M. Khera and S. Balguvhar, "Design of microcontroller based wireless SCADA system for real time data," UACEE Int. J. Adv. Electron. Eng., vol. 2, no. 1, 2011.

[8] Open Hub, “Android Language Breakdown,” 2017.

[9] Althos, "Bluetooth Tutorial," 2009. [Online]. Available: www.althos.com/tutorial/Bluetooth-tutorial.html. [Accessed: 01-Aug2018].

[10] General Instrument Corporation, "MOS Data," 1976

[11] General Instrument Corporation, “Data Catalog,” 1977. 